TRANSACTIONS OF THE

AMERICAN MATHEMATICAL SOCIETY

Volume 356, Number 2, Pages 557-571

S 0002-9947(03)03453-6

Article electronically published on September 22, 2003

\title{
TRUNCATED SECOND MAIN THEOREM WITH MOVING TARGETS
}

\author{
MIN RU AND JULIE TZU-YUEH WANG
}

\begin{abstract}
We prove a truncated Second Main Theorem for holomorphic curves intersecting a finite set of moving or fixed hyperplanes. The set of hyperplanes is assumed to be non-degenerate. Previously only general position or subgeneral position was considered.
\end{abstract}

\section{INTRODUCTION}

In this paper, we prove a truncated Second Main Theorem for holomorphic curves intersecting a finite set of moving or fixed hyperplanes. The set of hyperplanes is assumed to be non-degenerate (see the definition below). Previously only general position or subgeneral position was considered. Applications to the uniqueness problem appeared elsewhere (see [Ru2]). This paper is partially motivated by Ru's result (see Ru1]) that $\mathbb{P}^{n}-\mathcal{H}$ is Brody hyperbolic if and only $\mathcal{H}$ is non-degenerate, where $\mathcal{H}$ is a finite set of hyperplanes in $\mathbb{P}^{n}$.

To state our results, we first introduce some standard definitions in Nevanlinna theory. Let

$$
f=\left[f_{0}: \cdots: f_{n}\right]: \mathbb{C} \rightarrow \mathbb{P}^{n}(\mathbb{C})
$$

be a holomorphic map, where $f_{0}, \ldots, f_{n}$ are entire and without common zeros. Define $\mathbf{f}=\left(f_{0}, \ldots, f_{n}\right)$. $\mathbf{f}$ is called a reduced representation of $f$. The characteristic function $T_{f}(r)$ of $f$ is defined by

$$
T_{f}(r)=\int_{0}^{2 \pi} \log \left\|\mathbf{f}\left(r e^{i \theta}\right)\right\| \frac{d \theta}{2 \pi}-\log \|\mathbf{f}(0)\| .
$$

Note that the characteristic function $T_{f}(r)$ is independent of the choice of the reduced representation of $f$. A moving hyperplane assigns, to every $z \in \mathbb{C}$, a hyperplane given by

$$
H(z)=\left\{\left[x_{0}: \cdots: x_{n}\right] \in \mathbb{P}^{n}(\mathbb{C}) \mid \sum_{i=0}^{n} a_{i}(z) x_{i}=0\right\},
$$

where $a_{i}, 0 \leq i \leq n$, are entire functions without common zeros. Denote by $\mathbf{a}=\left(a_{0}, \ldots, a_{n}\right)$ the vector associated with $H$. A moving hyperplane $H$ gives a holomorphic map $\mathbb{P}(\mathbf{a}): \mathbb{C} \rightarrow \mathbb{P}^{n}(\mathbb{C})$. We define $T_{H}(r)=T_{\mathbb{P}(\mathbf{a})}(r)$.

Received by the editors January 17, 2001 and, in revised form, February 11, 2002.

2000 Mathematics Subject Classification. Primary 32H25, 32Q45.

The first author was supported in part by NSF grant DMS-9800361 and by NSA under grant number MDA904-01-1-0051, MSPF-02G-175.

The second author was supported in part by a NSC grant of Taiwan. 
We now define the counting function. For a moving hyperplane $H$, we say $(f, H)$ is free if $\mathbf{a} \cdot \mathbf{f} \not \equiv 0$, where $\mathbf{a}$ is the vector associated to $H$ and $\cdot$ is the dot product in $\mathbb{C}^{n+1}$. Under the assumption that $(f, H)$ is free, let $n_{f}(r, H)$ be the number of zeros of $\mathbf{a} \cdot \mathbf{f}$ in $|z|<r$. Let $n_{f}^{(n)}(r, H)$ be the number of zeros of $\mathbf{a} \cdot \mathbf{f}$ in $|z|<r$, where the multiplicity is counted only as $n$ if the vanishing order of $\mathbf{a} \cdot \mathbf{f}$ at the point is greater than or equal to $n$. The counting function is defined by

$$
N_{f}(r, H)=\int_{0}^{r} \frac{n_{f}(t, H)-n_{f}(0, H)}{t} d t+n_{f}(0, H) \log r,
$$

and the truncated counting function is

$$
N_{f}^{(n)}(r, H)=\int_{0}^{r} \frac{n_{f}^{(n)}(t, H)-n_{f}^{(n)}(0, H)}{t} d t+n_{f}^{(n)}(0, H) \log r .
$$

Denote by $\mathcal{M}$ the field of meromorphic functions on $\mathbb{C}$.

Definition 1.1. We say the set of moving hyperplanes $\mathcal{H}=\left\{H_{1}, \ldots, H_{q}\right\}$ (or $\left.\mathcal{A}=\left\{\mathbf{a}_{1}, \ldots, \mathbf{a}_{q}\right\}\right)$ is non-degenerate over $\mathcal{M}$ if $\operatorname{dim}(\mathcal{A})_{\mathcal{M}}=n+1$ and for each proper subset $\mathcal{A}_{1}$ of $\mathcal{A}$

$$
\left(\mathcal{A}_{1}\right)_{\mathcal{M}} \cap\left(\mathcal{A}-\mathcal{A}_{1}\right)_{\mathcal{M}} \cap \mathcal{A} \neq \emptyset
$$

where $(\mathcal{A})_{\mathcal{M}}$ is the linear span of $\mathcal{A}$ over the field $\mathcal{M}$.

Our result is stated as follows:

Theorem 1.1. Let $f: \mathbb{C} \rightarrow \mathbb{P}^{n}(\mathbb{C})$ be a holomorphic map. Let $\mathcal{H}=\left\{H_{1}, \ldots, H_{q}\right\}$ be a finite collection of moving hyperplanes. Assume that $\mathcal{H}$ is non-degenerate over $\mathcal{M}$, and $(f, H)$ is free for every $H \in \mathcal{H}$. Then

$$
T_{f}(r) \leq \sum_{i=1}^{q} n(2 n-1) N_{f}^{(n)}\left(r, H_{i}\right)+O\left(\max _{1 \leq i \leq q} T_{H_{i}}(r)\right)+O_{\text {exc }}\left(\log ^{+} T_{f}(r)\right),
$$

where $O_{\text {exc }}$ means the estimate holds except for $r$ in a set of finite Lebesgue measure.

Note that when $H_{1}, \ldots, H_{q}$ are (fixed) hyperplanes, we say that $\mathcal{H}=\left\{H_{1}, \ldots\right.$, $\left.H_{q}\right\}$ (or $\left.\mathcal{A}=\left\{\mathbf{a}_{1}, \ldots, \mathbf{a}_{q}\right\}\right)$ is non-degenerate over $\mathbb{C}$ if $\operatorname{dim}(\mathcal{A})=n+1$ and for each proper subset $\mathcal{A}_{1}$ of $\mathcal{A}$

$$
\left(\mathcal{A}_{1}\right) \cap\left(\mathcal{A}-\mathcal{A}_{1}\right) \cap \mathcal{A} \neq \emptyset,
$$

where $(\mathcal{A})$ is the linear span of $\mathcal{A}$ over $\mathbb{C}$. The proof of Theorem 1.1 implies that if $\mathcal{H}=\left\{H_{1}, \ldots, H_{q}\right\}$ is non-degenerate over $\mathbb{C}$, then every holomorphic map $f: \mathbb{C} \rightarrow \mathbb{P}^{n}(\mathbb{C})-\left(\bigcup_{j=1}^{q} H_{j}\right)$ must be constant. In this case we say that $P^{n}(\mathbb{C})-$ $\left(\bigcup_{j=1}^{q} H_{j}\right)$ is Brody hyperbolic. We note that Min Ru (cf. [Ru1]) proved that $P^{n}(\mathbb{C})-\left(\bigcup_{j=1}^{q} H_{j}\right)$ is Brody hyperbolic if and only if $\mathcal{H}=\left\{H_{1}, \ldots, H_{q}\right\}$ is nondegenerate over $\mathbb{C}$, so Theorem 1.1 can also be viewed as a quantitative extension of Ru's result.

Recall that (fixed) hyperplanes $\left\{H_{1}, \ldots, H_{q}\right\}$ (or $\left\{\mathbf{a}_{1}, \ldots, \mathbf{a}_{q}\right\}$ ) are said to be in general position if $\mathbf{a}_{\mu(0)}, \ldots, \mathbf{a}_{\mu(n)}$ are linearly independent for any injective map $\mu:\{0,1, \ldots, n\} \rightarrow\{1, \ldots, q\}$. Moving hyperplanes $\left\{H_{1}, \ldots, H_{q}\right\}$ are said to be in general position if $\left\{H_{1}(z), \ldots, H_{q}(z)\right\}$ are in general position for some (and hence for almost all) $z \in \mathbb{C}$. A typical example of $\mathcal{H}=\left\{H_{1}, \ldots, H_{q}\right\}$ being non-degenerate over $\mathcal{M}$ is that $\mathcal{H}$ is in general position. In this case, we have a stronger result. 
Theorem 1.2. Let $f: \mathbb{C} \rightarrow \mathbb{P}^{n}(\mathbb{C})$ be a holomorphic map. Let $\mathcal{H}=\left\{H_{1}, \ldots, H_{q}\right\}$ be a finite set of moving hyperplanes in general position. Assume that $(f, H)$ is free for every $H \in \mathcal{H}$. If $q \geq 2 n+1$, then

$$
\frac{q}{2 n+1} T_{f}(r) \leq \sum_{i=1}^{q} n N_{f}^{(n)}\left(r, H_{j}\right)+O\left(\max _{1 \leq i \leq q} T_{H_{i}}(r)\right)+O_{e x c}\left(\log ^{+} T_{f}(r)\right),
$$

where $O_{\text {exc }}$ means the estimate holds except for $r$ in a set of finite Lebesgue measure.

For the applications of the above truncated SMT with moving targets to the uniqueness problem, see $\mathrm{Ru} 2$. We also note that $\mathrm{Ru}$ and Stoll [R-S2] obtained the following inequality without truncation: Under the same assumptions as in Theorem 1.2, for every $\epsilon>0$, the inequality

$$
(q-2 n-\epsilon) T_{f}(r) \leq \sum_{i=1}^{q} N_{f}\left(r, H_{j}\right)+O\left(\max _{1 \leq i \leq q} T_{H_{i}}(r)\right)
$$

holds for all $r$ outside a set of finite Lebesgue measure.

\section{A REFINEMENT OF DIAGONAL EQUATIONS OF HOLOMORPHIC FUNCTIONS}

In this section, we give the following refinement for holomorphic functions satisfying a diagonal equation, which generalizes the well-known Borel Lemma in Nevanlinna theory. We use the standard notation in Nevanlinna theory.

Theorem 2.1. Let $f=\left[f_{0}: \cdots: f_{n}\right]: \mathbb{C} \rightarrow \mathbb{P}^{n}(\mathbb{C})$ be a holomorphic map, with $f_{0}, \ldots, f_{n}$ entire and no common zeros. Assume that $f_{n+1}$ is a holomorphic function and $f_{0}+\cdots+f_{n}+f_{n+1}=0$. If $\sum_{i \in I} f_{i} \neq 0$ for any proper subset $I$ of $\{0, \ldots$, $n+1\}$, then

$$
T_{f}(r) \leq \sum_{j=0}^{n+1} N_{f_{j}}^{(n)}(r, 0)+O_{e x c}\left(\log ^{+} T_{f}(r)\right)
$$

for $r \rightarrow \infty$, where $O_{\text {exc }}$ means the estimate holds except for $r$ in a set of finite Lebesgue measure.

To prove Theorem 2.1, we recall the following lemma from [B-M].

Lemma 2.2. Assume $\sum_{i=0}^{m} f_{i}=0$ but no non-empty proper subsum vanishes. If some proper subset of $\left\{f_{0}, \ldots, f_{m}\right\}$ is linearly dependent, then we can find an integer $l \geq 2$, a partition

$$
\{0,1, \ldots, m\}=I_{1} \cup \ldots \cup I_{l}
$$

into non-empty disjoint sets $I_{1}, \ldots, I_{l}$, and non-empty sets

$$
J_{1} \subseteq I_{1}, J_{2} \subseteq I_{1} \cup I_{2}, \ldots J_{l-1} \subseteq I_{1} \cup \ldots \cup I_{l-1}
$$

such that

$$
I_{1}, I_{2} \cup J_{1}, \ldots, I_{l} \cup J_{l-1}
$$

are minimal. Here, we say an index set $I \subset\{0,1, \ldots, m\}$ is minimal if the set $\left\{f_{i} \mid i \in I\right\}$ is linearly dependent, and for any proper subset $I^{\prime}$ of $I$ the set $\left\{f_{i} \mid i \in I^{\prime}\right\}$ is linearly independent. 
Proof. Throughout this proof, we use the term linear forms. Linear forms are the homogeneous polynomials of degree one in $m+1$ variables with coefficients in $\mathbb{C}$; that is, $L(X)=c_{0} x_{0}+\cdots+c_{m} x_{m}$, where $c_{0}, \ldots, c_{m} \in \mathbb{C}, X=\left(x_{0}, \ldots, x_{m}\right)$. We denote by $\mathcal{L}$ the set of linear forms which vanish on $\left(f_{0}, \ldots, f_{m}\right)$, so $L(X)=$ $c_{0} x_{0}+\cdots+c_{m} x_{m}$ is in $\mathcal{L}$ if and only if $c_{0} f_{0}+\cdots+c_{m} f_{m}=0$. By the assumption $f_{0}+\cdots+f_{m}=0, \mathcal{L}$ is non-empty. We make the following claim.

Claim 1. Every linear form $L$ in $\mathcal{L}$ can be written as

$$
L=\sum c_{J} L_{J} \text { with } L_{J} \in \mathcal{L}
$$

for certain minimal sets $J$, where $L_{J}$ is a linear combination of $\left\{x_{j} \mid j \in J\right\}$, and $c_{J}$ is constant.

We prove Claim 1 by induction on the length $t$ of $L$, i.e., the number of nonzero coefficients. The case $t=1$ is trivial. So assume that for some $t>1$ this holds for all elements of $\mathcal{L}$ of length strictly less than $t$. If $L \in \mathcal{L}$ has length exactly $t$, we may suppose that

$$
L=c_{0} x_{0}+\cdots+c_{t-1} x_{t-1}, \quad c_{i} \neq 0, \text { for } 0 \leq i \leq t-1 .
$$

If $I=\{0,1, \ldots, t-1\}$ is minimal, we are done. Otherwise, there is a linear form $L^{\prime}$ in $\mathcal{L}$ with less length. Without loss of generality we can assume that

$$
L^{\prime}=c_{0}^{\prime} x_{0}+\cdots+c_{k}^{\prime} x_{k}
$$

lies in $\mathcal{L}$ for some $k$ with $0 \leq k<t-1$ and $c_{0}^{\prime} \neq 0$. Then $L^{\prime}$ and $L^{\prime \prime}=c_{0}^{\prime} L-c_{0} L^{\prime}$ are both of length strictly less than $t$, and so the induction hypothesis can be applied to both linear forms. Since

$$
L=\left(c_{0} / c_{0}^{\prime}\right) L^{\prime}+\left(1 / c_{0}^{\prime}\right) L^{\prime \prime},
$$

$L$ has the desired decomposition. So Claim 1 is proved.

We now prove Claim 2.

Claim 2. Suppose $\sum_{i=0}^{m} f_{i}=0$ and $\sum_{i \in I} f_{i} \neq 0$ for some $I \subset N=\{0,1, \ldots, m\}$. Then there is a minimal set $J$ with $L_{J} \in \mathcal{L}$ such that $J \cap I \neq \emptyset$ and $J \cap I^{c} \neq \emptyset$, where $I^{c}$ is the complement of $I$ in $N$.

In fact, the set $L=\sum_{i=0}^{m} x_{i}$ is in $\mathcal{L}$ because $\sum_{i=0}^{m} f_{i}=0$. By Claim 1, we have

$$
L=\sum c_{J} L_{J} \text { with } L_{J} \in \mathcal{L}
$$

for certain minimal sets $J$. If Claim 2 is false, then every such $J$ is contained either in $I$ or in $I^{c}$. So $\sum_{i=0}^{m} x_{i}=L\left(x_{0}, \ldots, x_{m}\right)=\sum_{J \subset I} c_{J} L_{J}\left(x_{0}, \ldots, x_{m}\right)+$ $\sum_{J \subset I^{c}} c_{J} L_{J}\left(x_{0}, \ldots, x_{m}\right)$. However, for those $J \subset I, L_{J}\left(x_{0}, \ldots, x_{m}\right)$ involves only $\left\{x_{i} \mid i \in I\right\}$ while for those $J \subset I^{c}, L_{J}\left(x_{0}, \ldots, x_{m}\right)$ involves only $\left\{x_{i} \mid i \in I^{c}\right\}$. If we set $x_{i}=0$ for $i \in I^{c}$, the above equation becomes

$$
\sum_{i \in I} x_{i}=\sum_{J \subset I} c_{J} L_{J}\left(x_{0}, \ldots, x_{m}\right) .
$$

Since $L_{J} \in \mathcal{L}, L_{J}\left(f_{0}, \ldots, f_{m}\right)=0$. Hence $\sum_{i \in I} f_{i}=0$, which leads to a contradiction that proves Claim 2.

We now pick any minimal set $I_{1}$. By hypothesis $N=\{0,1, \ldots, m\}$ is not minimal, so $I_{1} \neq N$. Hence, $\sum_{i \in I_{1}} f_{i} \neq 0$. So Claim 2 implies that there exists a minimal set $I_{2}^{\prime}$ with $L_{I_{2}^{\prime}} \in \mathcal{L}$ such that $I_{2}^{\prime} \cap I_{1} \neq \emptyset$ and $I_{2}^{\prime} \cap I_{1}^{c} \neq \emptyset$, where $I_{1}^{c}$ is the 
complement of $I_{1}$ in $N$. Put $I_{2}=I_{2}^{\prime} \cap I_{1}^{c}$ and $J_{1}=I_{2}^{\prime} \cap I_{1}$. If $N=I_{1} \cup I_{2}$, then we are done. Otherwise, let $I=I_{1} \cup I_{2}$. Applying Claim 2 to $I$, there exists a minimal set $I_{3}^{\prime}$ with $L_{I_{3}^{\prime}} \in \mathcal{L}$, such that $I_{3}^{\prime} \cap I \neq \emptyset$ and $I_{3}^{\prime} \cap I^{c} \neq \emptyset$. Let $I_{3}=I_{2}^{\prime} \cap\left(I_{1} \cup I_{2}\right)^{c}$ and $J_{2}=I_{3}^{\prime} \cap\left(I_{1} \cup I_{2}\right)$. If $N=I_{1} \cup I_{2} \cup I_{3}$, then we are done. Otherwise, we repeat the same procedures until the union reaches $N$. This proves Lemma 2.2.

Proof of Theorem 2.1. If $f_{0}, \ldots, f_{n}$ are linearly independent, then this is a consequence of Cartan's truncated Second Main Theorem. If $f_{0}, \ldots, f_{n}$ are linearly dependent, then by Lemma 2.2 , we can find an integer $l \geq 2$, a partition

$$
\{0,1, \ldots, n+1\}=I_{1} \cup \ldots \cup I_{l}
$$

into non-empty disjoint sets $I_{1}, \ldots, I_{l}$, and non-empty sets

$$
J_{1} \subseteq I_{1}, J_{2} \subseteq I_{1} \cup I_{2}, \ldots, J_{l-1} \subseteq I_{1} \cup \ldots \cup I_{l-1}
$$

such that

$$
I_{1}, I_{2} \cup J_{1}, \ldots, I_{l} \cup J_{l-1}
$$

are minimal. Let $n_{i}=\# I_{i}$. Then $\sum_{i=1}^{l} n_{i}=n+2$. Without loss of generality we may assume that

$$
\left\{0, \ldots, n_{1}-1\right\}=I_{1},\left\{n_{1}, \ldots, n_{1}+n_{2}-1\right\}=I_{2}, \ldots,\left\{n+2-n_{l}, \ldots, n+1\right\}=I_{l} .
$$

We also write

$$
\hat{n}_{\lambda}=\sum_{\nu=1}^{\lambda} n_{\nu}
$$

Since $I_{1}$ is minimal, there is a linear relation among $\left\{f_{0}, \ldots, f_{n_{1}-1}\right\}$. That is,

$$
c_{0,1} f_{0}+\cdots+c_{n_{1}-1,1} f_{n_{1}-1}=\sum_{j \in I_{1}} c_{j, 1} f_{j}=0 .
$$

Define $c_{j, 1}=0$ for all $j \geq n_{1}$. Then

$$
\sum_{j=0}^{n+1} c_{j, 1} f_{j}=0
$$

Differentiation yields, for each positive integer $\rho$,

$$
\sum_{j=0}^{n+1} c_{j, 1} f_{j}^{(\rho)}=0
$$

Take $2 \leq \lambda \leq l$. Since $I_{\lambda} \cup J_{\lambda-1}$ is minimal, there are non-zero complex numbers $c_{j, \lambda}$ such that

$$
\sum_{j \in I_{\lambda} \cup J_{\lambda-1}} c_{j, \lambda} f_{j}=0 .
$$

Put $c_{j, \lambda}=0$ for all $j \notin\left(I_{\lambda} \cup J_{\lambda-1}\right)$. Then

$$
\sum_{j=0}^{n+1} c_{j, \lambda} f_{j}=0
$$

Differentiation yields, for each positive integer $\rho$,

$$
\sum_{j=0}^{n+1} c_{j, \lambda} f_{j}^{(\rho)}=0
$$


We consider an $(n+1) \times(n+2)$ master matrix $M$ given by

$$
M=\left[\begin{array}{ccc}
c_{0,1} f_{0} & \ldots & c_{n+1,1} f_{n+1} \\
c_{0,1} f_{0}^{\prime} & \cdots & c_{n+1,1} f_{n+1}^{\prime} \\
\vdots & \ddots & \vdots \\
c_{0,1} f_{0}^{\left(n_{1}-2\right)} & \ldots & c_{n+1,1} f_{n+1}^{\left(n_{1}-2\right)} \\
c_{0,2} f_{0} & \cdots & c_{n+1,2} f_{n+1} \\
\vdots & \ddots & \vdots \\
c_{0,2} f_{0}^{\left(n_{2}-1\right)} & \cdots & c_{n+1,2} f_{n+1}^{\left(n_{2}-1\right)} \\
c_{0,3} f_{0} & \cdots & c_{n+1,3} f_{n+1} \\
\vdots & \ddots & \vdots \\
c_{0,3} f_{0}^{\left(n_{3}-1\right)} & \cdots & c_{n+1,3} f_{n+1}^{\left(n_{3}-1\right)} \\
\vdots & \ddots & \vdots \\
c_{0, l} f_{0}^{\left(n_{l}-1\right)} & \cdots & c_{n+1, l} f_{n+1}^{\left(n_{l}-1\right)}
\end{array}\right],
$$

where we note that $n_{1}+\cdots+n_{l}=n+2$. We also note that, by (2.2) and (2.3), the sum of each row of $M$ is zero. Let $D_{j}$ be the determinant of the matrix obtained by deleting the $j$-th column of the master matrix $M$. Then, since the sum of each row of $M$ is zero, we actually have

$$
D_{j}=(-1)^{j} D_{0} .
$$

We now show that

$$
D_{0} \not \equiv 0 .
$$

For this, we first prove that

$$
D_{0}=\gamma_{1} \gamma_{2} \cdots \gamma_{l}
$$

where

and, for $2 \leq \lambda \leq l$,

$$
\gamma_{1}=\left|\begin{array}{ccc}
c_{1,1} f_{1} & \ldots & c_{n_{1}-1,1} f_{n_{1}-1} \\
\vdots & \ddots & \vdots \\
c_{1,1} f_{1}^{\left(n_{1}-2\right)} & \ldots & c_{n_{1}-1,1} f_{n_{1}-1}^{\left(n_{1}-2\right)}
\end{array}\right|
$$

$$
\gamma_{\lambda}=\left|\begin{array}{ccc}
c_{\hat{n}_{\lambda-1}, \lambda} f_{\hat{n}_{\lambda-1}} & \ldots & c_{\hat{n}_{\lambda}-1, \lambda} f_{\hat{n}_{\lambda}-1} \\
\vdots & \ddots & \vdots \\
c_{\hat{n}_{\lambda-1}, \lambda} f_{\hat{n}_{\lambda-1}}^{\left(n_{\lambda}-1\right)} & \ldots & c_{\hat{n}_{\lambda}-1, \lambda} f_{\hat{n}_{\lambda}-1}^{\left(n_{\lambda}-1\right)}
\end{array}\right|,
$$

where $\hat{n}_{\lambda}$ is defined in (2.1). (2.6) is true because of the definition of $D_{0}$ and the fact that $c_{j, 1}=0$ for $j \geq n_{1}$ and $c_{j, \lambda}=0$ for $j \geq \hat{n}_{\lambda}$ for $\lambda=2, \cdots, l$. Now, since $I_{1}$ is minimal, $c_{i, 1} \neq 0$ for $0 \leq i \leq n_{1}-1$, and also $\left\{f_{1}, \ldots, f_{n_{1}-1}\right\}$ is linearly independent, so $\gamma_{1} \neq \equiv 0$ by the property of the Wronskian. Also, since $I_{\lambda} \cup J_{\lambda-1}$ is minimal, $c_{i, \lambda} \neq 0$ for $\hat{n}_{\lambda-1} \leq i \leq \hat{n}_{\lambda}-1$ and also $\left\{f_{j}, j \in I_{\lambda}\right\}$ is linearly independent. So $\gamma_{\lambda} \not \equiv 0$ for $2 \leq \lambda \leq l$. Hence $D_{0} \not \equiv 0$ by (2.6). So (2.5) is verified. The rest of the proof is similar to the proof of Cartan's Second Main Theorem, replacing the Wronskian $W$ by $D_{0}$. The following is the detail. Consider the coordinate hyperplanes $H_{i}=\left\{\left[x_{0}: \cdots: x_{n}\right] \mid x_{i-1}=0\right\}$ for $1 \leq i \leq n+1$ and $H_{n+2}=\left\{\left[x_{0}: \cdots: x_{n}\right] \mid x_{0}+\cdots+x_{n}=0\right\}$, and notice that these hyperplanes are 
in general position. By the well-known "product to the sum formula" (see [Ru3], Lemma A3.1.6), we have

$$
\sum_{j=1}^{n+2} m_{f}\left(r, H_{j}\right) \leq \int_{0}^{2 \pi} \max _{0 \leq j \leq n+1} \log \frac{\left\|\mathbf{f}\left(r e^{i \theta}\right)\right\|^{n+1}}{\prod_{t=0, t \neq j}^{n+1}\left|f_{t}\left(r e^{i \theta}\right)\right|} \frac{d \theta}{2 \pi}+O(1) .
$$

However, using (2.4),

$$
\begin{aligned}
& \int_{0}^{2 \pi} \max _{0 \leq j \leq n+1} \log \frac{\left\|\mathbf{f}\left(r e^{i \theta}\right)\right\|^{n+1}}{\prod_{t=0, t \neq j}^{n+1}\left|f_{t}\left(r e^{i \theta}\right)\right|} \frac{d \theta}{2 \pi} \\
& =\int_{0}^{2 \pi} \max _{0 \leq j \leq n+1} \log \frac{\left|D_{0}\left(r e^{i \theta}\right)\right|}{\prod_{t=0, t \neq j}^{n+1}\left|f_{t}\left(r e^{i \theta}\right)\right|} \frac{d \theta}{2 \pi} \\
& +(n+1) \int_{0}^{2 \pi} \log || \mathbf{f}\left(r e^{i \theta}\right)|| \frac{d \theta}{2 \pi}-\int_{0}^{2 \pi} \log \left|D_{0}\left(r e^{i \theta}\right)\right| \frac{d \theta}{2 \pi} \\
& =\int_{0}^{2 \pi} \max _{0 \leq j \leq n+1} \log \frac{\left|D_{j}\left(r e^{i \theta}\right)\right|}{\prod_{t=0, t \neq j}^{n+1}\left|f_{t}\left(r e^{i \theta}\right)\right|} \frac{d \theta}{2 \pi}+(n+1) T_{f}(r)+O(1) \\
& -\int_{0}^{2 \pi} \log \left|D_{0}\left(r e^{i \theta}\right)\right| \frac{d \theta}{2 \pi} \\
& \leq \sum_{j=0}^{n+1} \int_{0}^{2 \pi} \log ^{+} \frac{\left|D_{j}\left(r e^{i \theta}\right)\right|}{\prod_{t=0, t \neq j}^{n+1}\left|f_{t}\left(r e^{i \theta}\right)\right|} \frac{d \theta}{2 \pi} \\
& +(n+1) T_{f}(r)-N_{D_{0}}(r, 0)+O(1)
\end{aligned}
$$

where, in the last step, we used the fact that, by Jensen's formula,

$$
\int_{0}^{2 \pi} \log \left|D_{0}\left(r e^{i \theta}\right)\right| \frac{d \theta}{2 \pi}=N_{D_{0}}(r, 0) .
$$

For each fixed $j$ with $0 \leq j \leq n+1$, we now estimate

$$
\int_{0}^{2 \pi} \log ^{+} \frac{\left|D_{j}\left(r e^{i \theta}\right)\right|}{\prod_{t=0, t \neq j}^{n+1}\left|f_{t}\left(r e^{i \theta}\right)\right|} \frac{d \theta}{2 \pi} .
$$

Note that $D_{j}$ does not involve $f_{j}$, so we write

$$
D_{j}=D\left(f_{0}, \ldots, f_{j-1}, f_{j+1}, \ldots, f_{n+1}\right) .
$$

Write $g_{i}=f_{i} / f_{j}$ for $1 \leq i \leq n+1$ and the fixed $j$. It is easy to verify that

$$
\begin{aligned}
& D\left(f_{0}, \ldots, f_{j-1}, f_{j+1}, \ldots, f_{n+1}\right) \\
& =f_{j}^{n+1} D\left(f_{0} / f_{j}, \ldots, f_{j-1} / f_{j}, f_{j+1} / f_{j}, \ldots, f_{n+1} / f_{j}\right) .
\end{aligned}
$$

In fact, from (2.6) we see that $D_{j}$ in fact is the product of several "small" Wronskians. So the above identity is true by the property of Wronskians. So

$$
D_{j}=f_{j}^{n+1} D\left(g_{0}, \ldots, g_{j-1}, g_{j+1}, \ldots, g_{n+1}\right) .
$$


Hence, by Theorem A1.2.5 in [Ru3] (the lemma of logarithmic derivatives),

$$
\begin{aligned}
& \int_{0}^{2 \pi} \log ^{+} \frac{\left|D_{j}\right|}{\left|f_{0} \cdots f_{j-1} f_{j+1} \cdots f_{n+1}\right|} \frac{d \theta}{2 \pi} \\
& =\int_{0}^{2 \pi} \log ^{+} \frac{\left|D\left(g_{0}, \ldots, g_{j-1}, g_{j+1}, \ldots, g_{n+1}\right)\right|}{\left|g_{0} \cdots g_{j-1} g_{j+1} \cdots g_{n+1}\right|} \frac{d \theta}{2 \pi} \\
& \leq O\left(\sum_{i=0}^{n+1} \log T_{g_{i}}(r)\right),
\end{aligned}
$$

where the inequality holds for all $r$ outside a set $E \subset(0,+\infty)$ with finite Lebesgue measure. Using the fact that $f_{0}+\cdots+f_{n}+f_{n+1}=0$, we get

$$
\sum_{i=0}^{n+1} \log T_{g_{i}}(r) \leq O\left(\log ^{+} T_{f}(r)\right) \text {. }
$$

Hence

$$
\int_{0}^{2 \pi} \log ^{+} \frac{\left|D_{j}\left(r e^{i \theta}\right)\right|}{\prod_{t=0, t \neq j}^{n+1}\left|f_{t}\left(r e^{i \theta}\right)\right|} \frac{d \theta}{2 \pi} \leq O\left(\log ^{+} T_{f}(r)\right),
$$

where the inequality holds for all $r$ outside a set $E \subset(0,+\infty)$ with finite Lebesgue measure. Hence, combining (2.7), (2.8) and (2.9),

$$
\sum_{j=1}^{n+2} m_{f}\left(r, H_{j}\right)+N_{D_{0}}(r, 0) \leq(n+1) T_{f}(r)+O\left(\log ^{+} T_{f}(r)\right),
$$

or we can write, by the First Main Theorem, the above inequality as

$$
T_{f}(r) \leq \sum_{j=1}^{n+2} N_{f}\left(r, H_{j}\right)-N_{D_{0}}(r, 0)+O\left(\log ^{+} T_{f}(r)\right) ;
$$

here the inequality holds for all $r$ outside a set $E \subset(0,+\infty)$ with finite Lebesgue measure. However, by the definition of $H_{j}$, we have

$$
N_{f}\left(r, H_{j}\right)=N_{f_{j-1}}(r, 0) .
$$

So the inequality

$$
T_{f}(r) \leq \sum_{j=0}^{n+1} N_{f_{j}}(r, 0)-N_{D_{0}}(r, 0)+O\left(\log ^{+} T_{f}(r)\right)
$$

holds for all $r$ outside a set $E \subset(0,+\infty)$ with finite Lebesgue measure. It remains to verify that

$$
\sum_{j=0}^{n+1} N_{f_{j}}(r, 0)-N_{D_{0}}(r, 0) \leq \sum_{j=0}^{n+1} N_{f_{j}}^{(n)}(r, 0) .
$$

Let $z_{0} \in \mathbb{C}$. Since $D_{j}=(-1)^{j} D_{0}$ and $f_{0}, \ldots, f_{n}$ have no common zeros, we may assume that $f_{0}\left(z_{0}\right) \neq 0, f_{j}$ vanishes at $z_{0}$ for $1 \leq j \leq q_{1}$ and $f_{j}$ does not vanish at $z_{0}$ for $j>q_{1}$. There are integers $k_{j} \geq 0$ and nowhere vanishing holomorphic functions $g_{j}$ in a neighborhood $U$ of $z_{0}$ such that

$$
f_{j}=\left(z-z_{0}\right)^{k_{j}} g_{j} \text { for } j=1, \ldots, n+1 .
$$


Here $k_{j}=0$ if $q_{1}<j \leq n+1$. Also we can assume that $k_{j} \geq n$ if $1 \leq j \leq q_{0}$ and $1 \leq k_{j}<n$, where $0 \leq q_{0} \leq q_{1}$. By the definition of $D_{0}$, we have

$$
D_{0}=\prod_{j=1}^{q_{0}}\left(z-z_{0}\right)^{k_{j}-n} h(z)
$$

where $h(z)$ is a holomorphic function defined on $U$. Thus $D_{0}$ vanishes at $z_{0}$ with order at least $\sum_{j=1}^{q_{0}}\left(k_{j}-n\right)=\sum_{j=1}^{q_{0}} k_{j}-q_{0} n$. Hence, we have

$$
\sum_{j=0}^{n+1} N_{f_{j}}(r, 0)-N_{D_{0}}(r, 0) \leq \sum_{j=0}^{n+1} N_{f_{j}}^{(n)}(r, 0)
$$

\section{Proof of Theorems 1.1 And 1.2}

In this section, we prove Theorems 1.1 and 1.2.

Proof of Theorem 1.1. Let $\mathcal{H}=\left\{H_{1}, \ldots, H_{q}\right\}$ be a finite set of moving hyperplanes. Assume that $\mathcal{H}$ is non-degenerate over $\mathcal{M}$; that is, (1.2) holds. Let

$$
H_{j}=\left\{\left[x_{0}: \cdots: x_{n}\right] \in \mathbb{P}^{n}(\mathbb{C}) \mid \sum_{i=0}^{n} a_{i j} x_{i}=0\right\},
$$

where $a_{i j}, 0 \leq i \leq n$, are entire functions without common zeros for each $1 \leq j \leq q$. For each $j$, there exists $j_{0}$ such that $a_{j_{0}, j} \not \equiv 0$. Let $b_{i j}=a_{i j} / a_{j_{0}, j}$. Then $b_{i j}$ are meromorphic function with the property that, for $1 \leq j \leq q, T_{b_{i j}}(r) \leq T_{H_{j}}(r)$. Let $\mathbf{a}_{j}=\left(b_{0 j}, \ldots, b_{n j}\right)$ and let $\mathcal{A}=\left\{\mathbf{a}_{1}, \ldots, \mathbf{a}_{q}\right\}$. Let $\left\{\mathbf{a}_{i_{1}}, \ldots, \mathbf{a}_{i_{m}}\right\}$ be a subset of $\mathcal{A}$. Suppose that this set is linearly dependent over $\mathcal{M}$ and no proper subset is linearly dependent over $\mathcal{M}$. Then we have a linear equation

$$
c_{i_{1}} \mathbf{a}_{i_{1}}+\cdots+c_{i_{m}} \mathbf{a}_{i_{m}}=0,
$$

where $c_{i_{j}}, 1 \leq j \leq m$, are nonzero meromorphic functions. By clearing the denominators, we can assume that $c_{i_{j}}$ are entire functions. We call (3.1) a minimal relation. Since $c_{i_{1}}, \ldots, c_{i_{m}}$ are determined by solving the system of linear equations $c_{i_{1}} b_{j, i_{1}}+\cdots+c_{i_{m}} b_{j, i_{m}}=0,0 \leq j \leq n$, they can be chosen as non-vanishing minors of the matrix with entries $b_{j, i_{\alpha}}, 1 \leq \alpha \leq m, 0 \leq j \leq n$, up to a sign. For such a choice of $c_{i_{\alpha}}, 1 \leq \alpha \leq m$, since $T_{b_{i j}}(r) \leq T_{H_{j}}(r)$, we have the following estimate:

$$
T_{c_{i_{\alpha}}}(r) \leq O\left(\max _{1 \leq i \leq q} T_{H_{i}}(r)\right) .
$$

Let $\mathcal{R}$ be the collection of all minimal relations associated to $\mathcal{A}$ arising in this way. We also note that $\mathbf{a}_{i}$ 's are pairwise linearly independent, because these hyperplanes are distinct. So we have $3 \leq m \leq n+2$.

Let $f=\left[f_{0}: \cdots: f_{n}\right]: \mathbb{C} \rightarrow \mathbb{P}^{n}(\mathbb{C})$ be a holomorphic map. Let $\mathbf{f}=\left(f_{0}, \ldots, f_{n}\right)$ be a reduced representation of $f$. We make the following claim:

Claim. There exist $n+1$ linearly independent vectors $\mathbf{a}_{i_{1}}, \ldots, \mathbf{a}_{i_{n+1}}$ in $\mathcal{A}$ such that

$$
\frac{T_{\mathbf{f} \cdot \mathbf{a}_{i_{\alpha}}}}{\mathbf{f} \cdot \mathbf{a}_{i_{1}}}(r) \leq \sum_{j=1}^{q}(2 n-1) N_{f}^{(n)}\left(r, H_{j}\right)+O\left(\max _{1 \leq i \leq q} T_{H_{i}}(r)\right)+O_{\text {exc }}\left(\log ^{+} T_{f}(r)\right)
$$

for $2 \leq \alpha \leq n+1$. 
To prove the claim, we first find a minimal relation in $\mathcal{R}$ containing $\mathbf{a}_{1}$. Without loss of generality, we assume that this minimal relation is

$$
c_{1} \mathbf{a}_{1}+c_{2} \mathbf{a}_{2}+\cdots+c_{m} \mathbf{a}_{m}=0 .
$$

Then $c_{1} \mathbf{f} \cdot \mathbf{a}_{1}+\cdots+c_{m} \mathbf{f} \cdot \mathbf{a}_{m}=0$. After rearranging the index again, we obtain an equation with no vanishing subsum:

$$
c_{1} \mathbf{f} \cdot \mathbf{a}_{1}+\cdots+c_{u} \mathbf{f} \cdot \mathbf{a}_{u}=0,
$$

$2 \leq u \leq n+2$. Theorem 2.1 and (3.2) thus imply

$$
\begin{aligned}
T_{\frac{c_{j} \mathbf{f} \cdot \mathbf{a}_{j}}{c_{1} \mathbf{f} \cdot \mathbf{a}_{1}}}(r) & \leq T_{\left[c_{1} \mathbf{f} \cdot \mathbf{a}_{1}: \ldots: c_{u} \mathbf{f} \cdot \mathbf{a}_{u}\right]}(r) \\
& \leq \sum_{t=1}^{u} N_{c_{t} \mathbf{f} \cdot \mathbf{a}_{t}}^{(u-2)}(r, 0)+O_{e x c}\left(\log ^{+} T_{\left[c_{1} \mathbf{f} \cdot \mathbf{a}_{1}: \ldots: c_{u} \cdot \mathbf{f} \cdot \mathbf{a}_{u}\right]}(r)\right) \\
& \leq \sum_{t=1}^{u} N_{\mathbf{f} \cdot \mathbf{a}_{t}}^{(u-2)}(r, 0)+O\left(\sum_{t=1}^{u} T_{c_{t}}(r)\right)+O_{e x c}\left(\log ^{+} T_{f}(r)\right) \\
& \leq \sum_{t=1}^{u} N_{\mathbf{f} \cdot \mathbf{a}_{t}}^{(u-2)}(r, 0)+O\left(\max _{1 \leq i \leq q} T_{H_{i}}(r)\right)+O_{e x c}\left(\log ^{+} T_{f}(r)\right),
\end{aligned}
$$

for $2 \leq j \leq u$.

From the definition of characteristic function,

$$
T_{\frac{\mathbf{f} \cdot \mathbf{a}_{j}}{\mathbf{f} \cdot \mathbf{a}_{1}}}(r) \leq T_{\frac{c_{j} \mathbf{f} \cdot \mathbf{a}_{j}}{c_{1} \mathbf{f} \cdot \mathbf{a}_{1}}}(r)+T_{\frac{c_{j}}{c_{1}}}(r) .
$$

Therefore the above inequalities and (3.2) imply that

$$
T_{\mathbf{f} \cdot \mathbf{a}_{j}}(r) \leq \sum_{t=1}^{q} N_{\mathbf{f} \cdot \mathbf{a}_{1}}^{(n) \mathbf{a}_{t}}(r, 0)+O\left(\max _{1 \leq i \leq q} T_{H_{i}}(r)\right)+O_{e x c}\left(\log ^{+} T_{f}(r)\right),
$$

for $2 \leq j \leq u$.

If the dimension of the vector space spanned by $\mathbf{a}_{1}, \ldots, \mathbf{a}_{u}$ over $\mathcal{M}$ is $n+1$, then we are done. Otherwise we assume that the dimension of the vector space spanned by $\mathbf{a}_{1}, \ldots, \mathbf{a}_{u}$ over $\mathcal{M}$ is less than $n+1$. Let $\mathcal{A}_{1}=\left\{\mathbf{a}_{i} \in \mathcal{A} \mid \mathbf{a}_{i} \in\left(\mathbf{a}_{1}, \ldots, \mathbf{a}_{u}\right)_{\mathcal{M}}\right\}$. Suppose that $\mathcal{A}_{1}=\left\{\mathbf{a}_{1}, \ldots, \mathbf{a}_{u_{1}}\right\}$. We now prove that

$$
T_{\mathbf{f} \cdot \mathbf{a}_{j}}(r) \leq \sum_{t=1}^{q} 2 N_{\mathbf{f} \cdot \mathbf{a}_{1}}^{(n)}(r) \mathbf{a}_{t}(r, 0)+O\left(\max _{1 \leq i \leq q} T_{H_{i}}(r)\right)+O_{e x c}\left(\log ^{+} T_{f}(r)\right),
$$

for $2 \leq j \leq u_{1}$. If $u_{1}=u$, then this is done already. Otherwise for each $u+1 \leq j \leq$ $u_{1}$ we have a minimal relation $c_{j} \mathbf{a}_{j}+c_{i_{1}} \mathbf{a}_{i_{1}}+\cdots+c_{i_{w}} \mathbf{a}_{i_{w}}=0$, where $\left\{i_{1}, \ldots, i_{w}\right\}$ is an index subset of $\{1, \ldots, u\}$. Repeating the procedure of deriving (3.5), we can show that

$$
T_{\substack{\mathbf{f} \cdot \mathbf{a}_{i_{j}} \\ \mathbf{f} \cdot \mathbf{a}_{j}}}(r) \leq \sum_{t=1}^{q} N_{\mathbf{f} \cdot \mathbf{a}_{t}}^{(n)}(r, 0)+O\left(\max _{1 \leq i \leq q} T_{H_{i}}(r)\right)+O_{e x c}\left(\log ^{+} T_{f}(r)\right),
$$

for some $1 \leq i_{j} \leq u$. Since

$$
\frac{\mathbf{f} \cdot \mathbf{a}_{j}}{\mathbf{f} \cdot \mathbf{a}_{1}}=\frac{\mathbf{f} \cdot \mathbf{a}_{j}}{\mathbf{f} \cdot \mathbf{a}_{i_{j}}} \frac{\mathbf{f} \cdot \mathbf{a}_{i_{j}}}{\mathbf{f} \cdot \mathbf{a}_{1}}
$$

its characteristic function satisfies (3.6). 
We now move to the second step of the proof. Since $\mathcal{H}$ is non-degenerate, $\left(\mathcal{A}_{1}\right)_{\mathcal{M}} \cap$ $\left(\mathcal{A}-\mathcal{A}_{1}\right)_{\mathcal{M}} \cap \mathcal{A} \neq \emptyset$. We can find an $\mathbf{a}_{i} \in\left(\mathcal{A}_{1}\right)_{\mathcal{M}} \cap\left(\mathcal{A}-\mathcal{A}_{1}\right)_{\mathcal{M}}$. From the definition of $\mathcal{A}_{1}$, we have $1 \leq i \leq u_{1}$. Since $\mathbf{a}_{i} \in\left(\mathcal{A}-\mathcal{A}_{1}\right)_{\mathcal{M}}$, after rearranging the linear forms we have a minimal relation $c_{i} \mathbf{a}_{i}+c_{u_{1}+1} \mathbf{a}_{u_{1}+1}+\cdots+c_{w} \mathbf{a}_{w}=0$. Similarly, after rearranging the index, we have an equation with no vanishing proper subsum

$$
c_{i} \mathbf{a}_{i}+c_{u_{1}+1} \mathbf{a}_{u_{1}+1}+\cdots+c_{\nu} \mathbf{a}_{\nu}=0, \quad \nu \leq w .
$$

Therefore, similarly to the derivation of (3.5),

$$
T_{\frac{\mathbf{f} \cdot \mathbf{a}_{u_{1}+1}}{\mathbf{f} \cdot \mathbf{a}_{i}}}(r) \leq \sum_{t=1}^{q} N_{\mathbf{f} \cdot \mathbf{a}_{t}}^{(n)}(r, 0)+O\left(\max _{1 \leq i \leq q} T_{H_{i}}(r)\right)+O_{e x c}\left(\log ^{+} T_{f}(r)\right) .
$$

Since

$$
\frac{\mathbf{f} \cdot \mathbf{a}_{u_{1}+1}}{\mathbf{f} \cdot \mathbf{a}_{1}}=\frac{\mathbf{f} \cdot \mathbf{a}_{u_{1}+1}}{\mathbf{f} \cdot \mathbf{a}_{i}} \frac{\mathbf{f} \cdot \mathbf{a}_{i}}{\mathbf{f} \cdot \mathbf{a}_{1}}
$$

from (3.6) and (3.8) we have

$$
\frac{T_{\mathbf{f} \cdot \mathbf{a}_{u_{1}+1}}}{\mathbf{f} \cdot \mathbf{a}_{1}}(r) \leq \sum_{t=1}^{q} 3 N_{\mathbf{f} \cdot \mathbf{a}_{t}}^{(n)}(r, 0)+O\left(\max _{1 \leq i \leq q} T_{H_{i}}(r)\right)+O_{e x c}\left(\log ^{+} T_{f}(r)\right),
$$

If $\operatorname{dim}\left(L_{1}, \ldots, L_{u_{1}+1}\right)_{\mathcal{M}}=n+1$, then we are done. Otherwise, we can repeat the same argument. Then we will obtain a sequence of collections of linear forms $\mathcal{A}_{1}, \ldots, \mathcal{A}_{r}$ such that $\operatorname{dim}\left(\mathcal{A}_{1}\right)_{\mathcal{M}}<\operatorname{dim}\left(\mathcal{A}_{2}\right)_{\mathcal{M}}<\cdots<\operatorname{dim}\left(\mathcal{A}_{r}\right)_{\mathcal{M}}=n+1$ and

$$
T_{\mathbf{f} \cdot \mathbf{a}}(r) \leq \sum_{t=1}^{q} 2 i N_{\mathbf{f} \cdot \mathbf{a}_{1}}^{(n)}(r, 0)+O\left(\operatorname{axp}_{1 \leq i \leq q} T_{H_{i}}(r)\right)+O_{e x c}\left(\log ^{+} T_{f}(r)\right),
$$

for $\mathbf{a} \in \mathcal{A}_{i}$. Since $\operatorname{dim}(\mathcal{A})_{\mathcal{M}}=n+1$ and the cardinality of $\mathcal{A}_{1}$ is at least $2, r \leq n$. It's also clear from the proof that to show the claim we only need to show (3.10) up to $r-1$, and show an inequality similar to (3.8) for one element in $\mathcal{A}_{r}-\mathcal{A}_{r-1}$. Hence we arrive at (3.3). Thus the claim is proved.

By the claim, there are $n+1$ linearly independent vectors $\mathbf{a}_{i_{1}}, \ldots, \mathbf{a}_{i_{n+1}} \in \mathcal{A}$ such that (3.3) holds. Hence

$$
\begin{aligned}
T_{f}(r) & \leq \sum_{j=2}^{n+1} T_{\mathbf{f} \cdot \mathbf{a}_{i_{j}}}(r)+O\left(\max _{1 \leq i \leq q} T_{H_{i}}(r)\right) \\
& \leq \sum_{t=1}^{q} n(2 n-1) N_{\mathbf{f} \cdot \mathbf{a}_{t}}^{(n)}(r, 0)+O\left(\max _{1 \leq i \leq q} T_{H_{i}}(r)\right)+O_{e x c}\left(\log ^{+} T_{f}(r)\right) .
\end{aligned}
$$

This finishes the proof of Theorem 1.1.

Proof of Theorem 1.2. Let

$$
H_{j}=\left\{\left[x_{0}: \cdots: x_{n}\right] \in \mathbb{P}^{n}(\mathbb{C}) \mid \sum_{i=0}^{n} a_{i j} x_{i}=0\right\},
$$

where $a_{i j}, 0 \leq i \leq n$, are entire functions without common zeros for each $1 \leq j \leq q$. For each $j$, there exists $j_{0}$ such that $a_{j_{0}, j} \not \equiv 0$. Let $b_{i j}=a_{i j} / a_{j_{0}, j}$. Then $b_{i j}$ are meromorphic functions with the property that, for $1 \leq j \leq q, T_{b_{i j}}(r) \leq T_{H_{j}}(r)$. 
Let $\mathbf{a}_{j}=\left(b_{0 j}, \ldots, b_{n j}\right)$. Let $I \subset\{2, \ldots, q\}$ be the index set with the property that $i \in I$ if and only if

$$
T_{\mathbf{f} \cdot \mathbf{a}_{i}}(r) \leq \sum_{i=1}^{q} N_{\mathbf{f} \cdot \mathbf{a}_{1}}^{(n) \mathbf{a}_{t}}(r, 0)+O\left(\max _{1 \leq i \leq q} T_{H_{i}}(r)\right)+O_{e x c}\left(\log ^{+} T_{f}(r)\right) .
$$

We first show that $\# I \geq n+1$. After rearranging the index, we assume that $I=\{2, \ldots, u\}$, and $u \leq n$. For dimensional reasons, $\left\{\mathbf{a}_{1}, \mathbf{a}_{n+1}, \ldots, \mathbf{a}_{2 n+1}\right\}$ is always linearly dependent over $\mathcal{M}$, i.e.

$$
c_{1} \mathbf{a}_{1}+c_{n+1} \mathbf{a}_{n+1}+\cdots+c_{2 n+1} \mathbf{a}_{2 n+1}=0 .
$$

Moreover, since these linear forms are in general position, we can solve for $c_{1}, c_{n+1}$, $\ldots, c_{n+2}$ explicitly. In fact, let

$$
A=\left(\begin{array}{ccc}
a_{10} & \ldots & a_{1 n} \\
a_{n+1,0} & \ldots & a_{n+1, n} \\
\vdots & \ddots & \vdots \\
a_{2 n+1,0} & \ldots & a_{2 n+1, n}
\end{array}\right),
$$

and let $(-1)^{i-1} A_{i}$ be the determinant of the matrix obtained by deleting the i-th row, $1 \leq i \leq n+2$, from $A$; then $c_{1}=A_{1}, c_{n+1}=A_{2}, \ldots, c_{2 n+1}=A_{n+2}$. For such $c_{1}, c_{n+1}, \ldots, c_{2 n+1}$, since $T_{b_{i j}}(r) \leq T_{H_{j}}(r)$, we have

$$
T_{c_{1}}(r) \leq O\left(\max _{1 \leq i \leq q} T_{H_{i}}(r)\right)
$$

and, for $n+1 \leq j \leq 2 n+1$,

$$
T_{c_{j}}(r) \leq O\left(\max _{1 \leq i \leq q} T_{H_{i}}(r)\right) .
$$

After rearranging the index we will have an equation

$$
c_{1} \mathbf{f} \cdot \mathbf{a}_{1}+c_{n+1} \mathbf{f} \cdot \mathbf{a}_{n+1}+\cdots+c_{w} \mathbf{f} \cdot \mathbf{a}_{w}=0,
$$

with no proper subsum vanishing. Therefore, similarly to (3.5), we conclude that

$$
T_{\frac{\mathbf{f} \cdot \mathbf{a}_{n+1}}{\mathbf{f} \cdot \mathbf{a}_{1}}}(r) \leq \sum_{t=1}^{q} N_{\mathbf{f} \cdot \mathbf{a}_{t}}^{(n)}(r, 0)+O\left(\max _{1 \leq i \leq q} T_{H_{i}}(r)\right)+O_{e x c}\left(\log ^{+} T_{f}(r)\right) .
$$

This contradicts the fact that $n+1$ is not in $I$. Thus $\# I \geq n+1$. By the "in general position" assumption, any $n+1$ hyperplanes in $\mathcal{H}$ are linearly independent. Therefore, similarly to (3.11), we can derive the following inequality:

$$
T_{f}(r) \leq \sum_{j=1}^{q} n N_{f}^{(n)}\left(r, H_{j}\right)+O\left(\max _{1 \leq i \leq q} T_{H_{i}}(r)\right)+O_{e x c}\left(\log ^{+} T_{f}(r)\right) .
$$

We now deduce the inequality of the theorem by induction on $q$. Let $\mathcal{H}_{\gamma}$ be a subset of $\mathcal{H}$ consisting of $\gamma \geq 2 n+1$ elements. When $\gamma=2 n+1$, this is done by 
(3.12). By the induction assumption

$$
\frac{\gamma}{2 n+1} T_{f}(r) \leq \sum_{H \in \mathcal{H}_{\gamma}} n N_{f}^{(n)}(r, H)+O\left(\max _{1 \leq i \leq q} T_{H_{i}}(r)\right)+O_{e x c}\left(\log ^{+} T_{f}(r)\right)
$$

for any subset $\mathcal{H}_{\gamma}$ of $\mathcal{H}$ consisting of $\gamma \geq 2 n+1$ elements. For $\mathcal{H}_{\gamma+1}$, we can choose $\gamma$ linear forms at a time and apply (3.13). This gives $\gamma+1$ inequalities like (3.13). Summing up these $\gamma+1$ inequalities, we have

$$
\frac{\gamma+1}{2 n+1} T_{f}(r) \leq \sum_{j=1}^{q} n N_{f}^{(n)}\left(r, H_{j}\right)+O\left(\max _{1 \leq i \leq q} T_{H_{i}}(r)\right)+O_{e x c}\left(\log ^{+} T_{f}(r)\right) .
$$

This completes the proof of Theorem 1.2.

\section{Some Results on ABC variety}

Motivated by Theorem 1.1, we introduce the concept of ABC variety. The definition is similar to the concept introduced by Buium (cf. [Bu]) in the function field case. For more discussions in this direction, see [W]. Let $V$ be a smooth complex projective variety. Let $A$ be an ample divisor on $X$. The characteristic (or height) function of $f$ with respect to $A$ is defined by

$$
T_{A}(r, f)=\int_{0}^{r} \int_{\triangle_{t}} f^{*} c_{1}(A) \frac{d t}{t}
$$

where $c_{1}(A)$ is the first Chern form of the line bundle $[A]$ associated with $A$. Since, with respect to different ample divisors, the characteristic functions of $f$ differ only by a constant multiple plus a bounded term, we denote $T_{A}(r, f)$ simply by $T_{f}(r)$ for some ample divisor $A$. Let $D$ be an effective divisor. The proximity function $m_{f}(r, D)$ is defined by

$$
m_{f}(r, D)=\int_{0}^{2 \pi} \log \frac{1}{\left\|s\left(f\left(r e^{i \theta}\right)\right)\right\|} \frac{d \theta}{2 \pi}
$$

where $s$ is the canonical section of $[D]$. The truncated counting function $N_{f}^{(n)}(r, D)$ of $f$ is the same as what we defined in Section 1.

Definition. Let $V$ be a smooth projective variety defined over $\mathbb{C}$ of dimension $n$. Let $D$ be an effective divisor over $V$. The pair $(V, D)$ is called an ABC-variety if there is a positive constant $C$ such that for all holomorphic map $f: \mathbb{C} \rightarrow V$,

$$
T_{f}(r) \leq C N_{f}^{(n)}(r, D)+O_{e x c}\left(\log ^{+} T_{f}(r)\right) .
$$

Theorem 1.1 implies that $\left(\mathbb{P}^{n}(\mathbb{C}), \bigcup_{H \in \mathcal{H}} H\right)$ is an $A B C$-variety if $\mathcal{H}$ is nondegenerate. We now prove that they are in fact equivalent.

Theorem 4.1. Let $\mathcal{H}$ be a finite set of hyperplanes in $\mathbb{P}^{n}(\mathbb{C})$. Then

$$
\left(\mathbb{P}^{n}(\mathbb{C}), \bigcup_{H \in \mathcal{H}} H\right)
$$

is an $A B C$-variety if and only if $\mathcal{H}$ is non-degenerate over $\mathbb{C}$. Or equivalently, $\left(\mathbb{P}^{n}(\mathbb{C}), \bigcup_{H \in \mathcal{H}} H\right)$ is an $A B C$-variety if and only if $\mathbb{P}^{n}(\mathbb{C})-\bigcup_{H \in \mathcal{H}} H$ is Brody hyperbolic. 
In fact, if $\left(\mathbb{P}^{n}(\mathbb{C}), \bigcup_{H \in \mathcal{H}} H\right)$ is an $A B C$-variety, then (4.1) holds, so it implies that $\mathbb{P}^{n}(\mathbb{C})-\bigcup_{H \in \mathcal{H}} H$ is Brody hyperbolic. By the result of $[\mathrm{Ru1}], \mathbb{P}^{n}(\mathbb{C})-\bigcup_{H \in \mathcal{H}} H$ is Brody hyperbolic if and only if $\mathcal{H}$ is non-degenerate over $\mathbb{C}$. This, together with Theorem 1.1, implies Theorem 4.1. Below, for completeness, we include a proof which contains the step that explains why $\mathbb{P}^{n}(\mathbb{C})-\bigcup_{H \in \mathcal{H}} H$ Brody hyperbolic implies $\mathcal{H}$ non-degenerate.

Proof of Theorem 4.1. As we indicated, we only need to prove that if $\mathbb{P}^{n}(\mathbb{C})$ $\bigcup_{H \in \mathcal{H}} H$ is an $A B C$-variety, then $\mathcal{H}$ is non-degenerate. To prove this, we first recall a result from [Ru1].

Proposition $(\mathrm{Ru}) . \mathcal{H}$ is non-degenerate over $\mathbb{C}$ if and only if for every $\mathcal{H}$-admissible subspace $V$ of $\mathbb{P}^{n}(\mathbb{C})$ of projective dimension greater than or equal to one, $\mathcal{H} \cap V$ contains at least three distinct hyperplanes which are linearly dependent over $F$, where $V$ is called $\mathcal{H}$-admissible if $V$ is not contained in any hyperplane in $\mathcal{H}$.

Assume that $\mathcal{H}$ is degenerate over $\mathbb{C}$. Then the above proposition implies that there exists an $\mathcal{H}$-admissible subspace $V$ of $\mathbb{P}^{n}$ of projective dimension greater than or equal to 1 such that $\mathcal{H} \cap V$ does not contain at least three distinct hyperplanes which are linearly dependent over $\mathbb{C}$. After a linear change of basis we may assume that $V=\mathbb{P}^{m}, m \leq n$. Then $\mathcal{H} \cap V$ contains exactly $q$ distinct hyperplanes which are linearly independent over $\mathbb{C}$, and $q \leq n+1$. Obviously there is a non-constant holomorphic map $f: \mathbb{C} \rightarrow \mathbb{P}^{n}(\mathbb{C})$ which omits these coordinate hyperplanes. On the other hand, by our assumption, $\left(\mathbb{P}^{n}(\mathbb{C}), \bigcup_{H \in \mathcal{H}} H\right)$ is an $A B C$-variety; thus (4.1) holds for $D=\bigcup_{H \in \mathcal{H}} H$. This implies that $f$ must be constant. This leads to a contradiction.

Finally, we conjecture that $(V, D)$ is an $A B C$-variety if and only if $V-D$ is Kobayashi hyperbolic.

\section{ACKNOWLEDGMENTS}

The first author wishes to thank the Institute of Mathematics, Academia Sinica, Taiwan, for kind hospitality and financial support while part of the work on this paper took place.

\section{REFERENCES}

[B-M] Brownawell, W.D. and Masser, D.W.: Vanishing sums in function fields. Math. Proc. Comb. Phil. Soc. 100, 427-434 (1986). MR 87k:11080

[Bu] Buium, A.: The abc theorem for abelian varieties. International Math. Research Notices 5, 219-233 (1994). MR 95c:11074

[L] Lang, S.: Introduction to complex hyperbolic spaces. New York Berlin Heidelberg: Springer 1987. MR 88f:32065

[Ru1] Ru, M.: Geometric and arithmetic aspects of $P^{n}$ minus hyperplanes. American Journal of Mathematics, 117, 307-321 (1995). MR 97c:32031

[Ru2] Ru, M.: A uniqueness theorem for moving targets without counting multiplicities. Proc. Amer. Math. Soc., 129, 2701-2707 (2000). MR 2002e:32024

[Ru3] Ru, M.: Nevanlinna theory and its relation to Diophantine approximation. River Edge, New Jersey: World Scientific Pub., (2001). MR 2002g:11106

[R-S1] Ru, M. and Stoll, W.: The second main theorem for moving targets. J. Geom. Anal. 1, 99-138 (1991). MR 92j:32098

[R-S2] Ru, M. and Stoll, W.: The Cartan conjecture for moving targets. In: Several Complex Variables and Complex Geometry, Part 2 (Proc. Sympos. Pure Math., vol. 52, pp. 99-138) Providence, Rhode Island: Amer. Math. Soc. 1991. MR 93f:32028 
[S] Steinmetz, N.: Eine Verallgemeinerung des zweiten Nevanlinnaschen Hauptsatzes. J. Reine Angew. Mathematik 368, 134-141 (1985). MR 87i:30056

[W] Wang, J. T-Y.: abc estimate, integral points, and geometry of $P^{n}$ minus hyperplanes. Mathematical Research Letters 6, 357-370 (1999). MR 2000j:11114

Department of Mathematics, University of Houston, Houston, Texas 77204

E-mail address: minru@math.uh.edu

Institute of Mathematics, Academia Sinica, Nankang, Taipei 11529 Taiwan, Republic OF CHINA

E-mail address: jwang@math.sinica.edu.tw 